\title{
ON HYSTERESIS OF ION CHANNELS
}

\author{
CAN E. KORMAN ${ }^{1, *}$ AND IsAaK D. MAYERGOYZ ${ }^{2}$
}

\begin{abstract}
Ion channel proteins have many conformational (metastable) states and, for this reason, they exhibit hysteresis. This fact is responsible for the non-Markovian stochastic nature of single ion channel recordings. It is suggested in the paper that the stochastic single channel recordings can be modeled as the random outputs of rectangular hysteresis loops driven by stochastic processes. The latter problem can be mathematically treated as an exit problem for stochastic processes or by using the theory of stochastic processes on graphs. It is also demonstrated in the paper that the collective action of sodium and potassium channels responsible for the generation and propagation of action potentials exhibit hysteresis. This demonstration is accomplished by using the inverse problem approach to the nonlinear Hodgkin-Huxley diffusion equation.
\end{abstract}

Mathematics Subject Classification. 92B05, 92C20, 35Q92, 60G20.

Received December 21, 2018. Accepted November 26, 2019.

\section{INTRODUCTION}

Neurons are the main building components of the brain and the nervous system. They form complex networks for the purposes of memory formation, motor control, processing of sensory input, etc. Neurons are characterized by their diversity adapted to the vast range of functions. In the human brain alone, it is estimated that there are about 100 billion neurons, and several orders of magnitude more neuronal connections. To make the paper accessible to a broader audience, we present in Appendix A a brief overview of the biological structure of neurons. The detailed discussion of these and many other important facts can be found in numerous books on neural science [1-6]. This list of references is not exhaustive but rather suggestive.

From a physics point of view, neurons are highly complex and intricate electric systems. Ion channels of neurons are at the very foundation of the functioning of these systems. The ion channels are ubiquitous. They are present in somas, axons, and dendrites of neural cells. These channels are crucial for establishing and maintaining the membrane voltage, for generation and propagation of action potentials and for strengthening or weakening of synaptic connections between neurons. Ion channels are formed by specialized proteins embedded in phospholipid bilayers of membranes. A schematic picture of an embedded ion channel is shown in Figure 1.

Generally, ion channels are composed of several protein subunits which under certain conditions form pores. These pores can be viewed as narrow salt-water filled tunnels that permit specific ions to pass through. Ions travel through channels down gradients of electrochemical potentials. These potential gradients have two distinct components: the diffusion component due to ion concentration gradients and drift component due to the presence

Keywords and phrases: Ion channels, hysteresis, stochastic processes, Preisach model.

1 Department of Electrical and Computer Engineering, George Washington University, Washington, DC 20052, USA.

2 Department of Electrical and Computer Engineering, University of Maryland, College Park, MD 20742, USA.

* Corresponding author: korman@gwu.edu

(C) The authors. Published by EDP Sciences, 2020

This is an Open Access article distributed under the terms of the Creative Commons Attribution License (http://creativecommons.org/licenses/by/4.0), which permits unrestricted use, distribution, and reproduction in any medium, provided the original work is properly cited. 


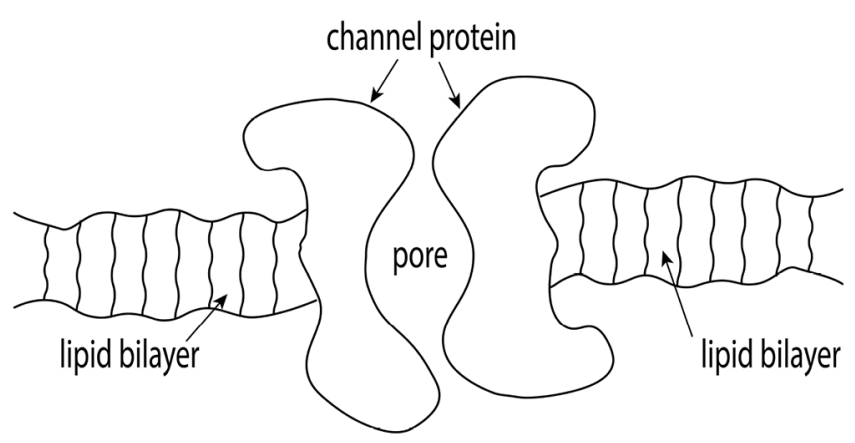

Figure 1. Schematic of an embedded ion channel.

of electric fields (i.e. electric potential gradients). The rate of ion passage through channels, is quite high and may reach about one million ions per second or higher. Nevertheless, ion currents through single channels are quite small, and they are usually are on the order of picoamperes. The structures of ion channels are discussed in detail in the classical book of Hille [12]. It must be remarked that ion channel structures and their functioning are only partly understood. For this reason, ion channels are currently a subject of extensive research in neural science.

There exists a large variety of ion channels. Ion channels can be classified by gating, i.e. by actions responsible for opening and closing these channels. In this respect, there are voltage-gated and ligand-gated ion channels. The performance of voltage- gated channels is controlled by potential difference (voltage) across the membrane in the locations of the channels, whereas the performance of ligand-gated channels is controlled by chemical signals produced by the binding of ligands (i.e., special stimulatory molecules) to channels.

Ion channels can also be classified by the type of ions that are allowed to selectively pass through channels. In this respect, there are four types of ion channels that are most important for the functioning of neurons. They are sodium $\left(\mathrm{Na}^{+}\right)$channels, potassium $\left(\mathrm{K}^{+}\right)$channels, calcium $\left(\mathrm{Ca}^{2+}\right)$ channels and chloride $\left(\mathrm{Cl}^{-}\right)$channels. Voltage-gated sodium and potassium channels are instrumental in the generation, formation, and propagation of action potentials. Whereas ligand-gated channels play the central role in the formation and functioning of synaptic connections between neurons.

Next, we consider the principle of operation of ion channels. As discussed before, proteins are the "walls" of ion channels. Proteins usually have a very large number of metastable states corresponding to different local minima of free energy separated by some energy barriers. In biophysics and neural science, these metastable states are called conformational states. Ion channel functioning depends on these conformational states. This is because proteins assume different geometric shapes in different conformational states. In some of these conformational states, proteins may form pores that permit specific ions to pass through. On the other hand, protein conformational states with the absence of sufficiently wide pores can be prohibitive to the passage to specific ions. The transition between these protein conformational states may be triggered by changes in local membrane voltage in voltage-gated channels.

This transition may also be caused by binding of special stimulating molecules in ligand-gated channels. It is quite possible that there is also another physical mechanism of switching of protein conformational states. This mechanism is related to ion flow through channels, and it was studied by Chinarov and his colleagues [2]. Indeed, the electric fields of ions at very short distances within narrow channel pore confinements may be comparable and even higher than the electric fields produced by voltage gating. Furthermore, ions traveling through channel pores may bind at some sites inside these pores. The electrostatic fields of these bound ions may also exceed the electric fields produced by voltage gating. The presented discussion suggests that ion currents through channels may result in the changes in protein conformational states leading to subsequent changes in the functioning of ion channels. 


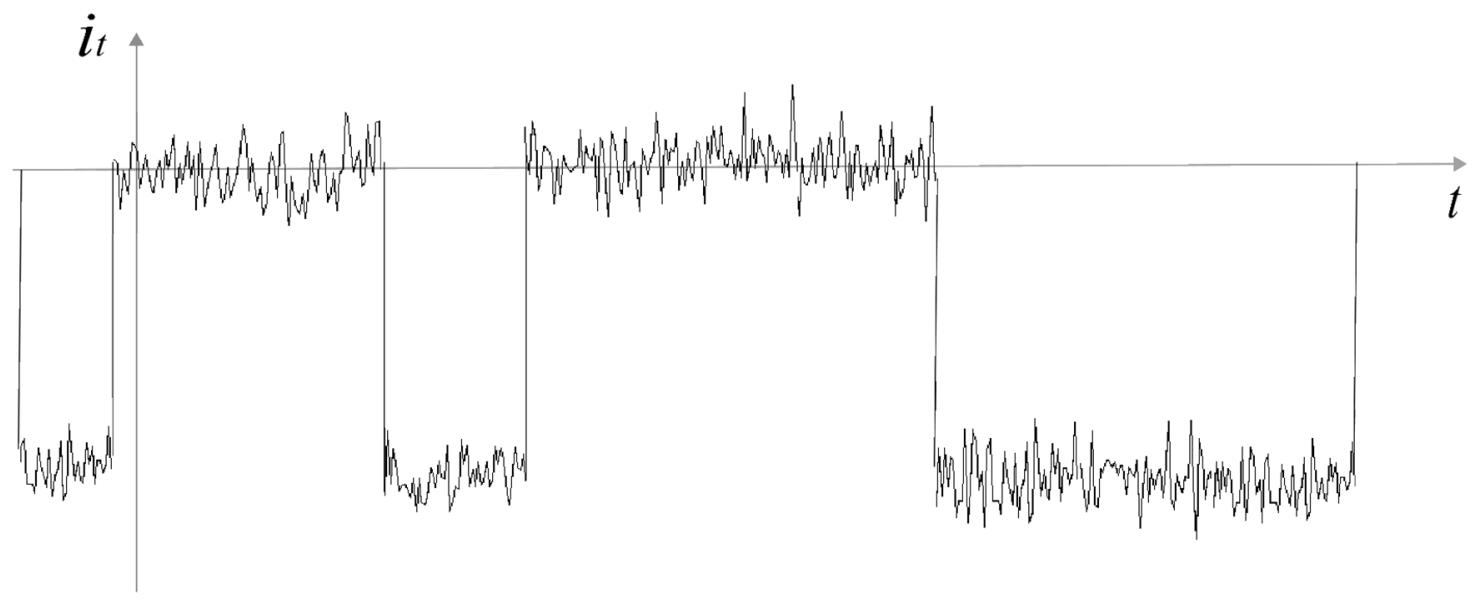

Figure 2. Schematic of an embedded ion channel.

\section{ION CHANNELS}

As discussed before, channel proteins have large numbers of conformational (i.e. metastable) states. For this reason, electrical properties of ion channels exhibit hysteresis. This hysteresis has been already observed experimentally and studied theoretically. Some sample publications in this area are [9-14], and the number of research reports related to hysteresis of ion channels is growing. The existence of hysteresis of ion channels implies that ion channels are endowed with memory. This memory is encoded in conformational states of ion channel proteins. This means that the past history of the conformational states of ion channels may alter their future conformational states and, consequently, their functioning. As argued before, currents through ion channels may affect conformational states of their proteins and, consequently, encode new information. In other words, the past history of ion currents through channels may affect their future functioning. Historically, ion channels were difficult to detect experimentally because of tiny currents through these channels. This difficulty was by and large removed through the development of the patch-clamp technique. This technique was introduced by Erwin Neher and Bert Sakmann in the late 1970s [20]. The patch-clamp technique made the recording of single ion channels possible, and it became one of the principal sources of information about these channels. Neher and Sakmann were awarded the Nobel Prize in 1991 for their work in this area. There are numerous patch-clamp techniques for both voltage and current controlled measurements, as well as ligand-controlled measurements. In the patch-clamp technique, a hollow glass micropipette with a very narrow tip of diameter in micrometer range is pressed against a cell membrane and suction is subsequently applied to form a tight sealing around the edge of the tip. The micropipette is filled with an electrolyte solution which matches the ionic composition of the electrolyte solution in the exterior of neurons. This pipette and specially designed electronic circuitry with amplifiers are used to record ion currents. A schematic representation of such recording of ion current through a single channel is shown in Figure 2.

It is apparent from the above figure that ion currents through single channels are stochastic in nature. In other words, channels randomly switch between open and closed conformations. It has been observed that random ion current recordings are modified by applied membrane voltage in the case of voltage-gated channels and by ligand bindings in the case of ligand-gated channels. It is most likely that the randomness of channel currents is caused by thermal noise, although other sources of noise may be present as well. In the noisy environment, protein states and their geometric configurations are not static. Proteins execute random motions around their conformational configurations. These random motions occur within energy wells with energy minima corresponding to metastable (i.e. conformational) states. When sufficiently large deviations (i.e. large energy kicks) of noise are realized, they may move proteins over energy barriers into adjacent energy wells corresponding to other conformational states. This is a possible physical mechanism of random switching between protein 


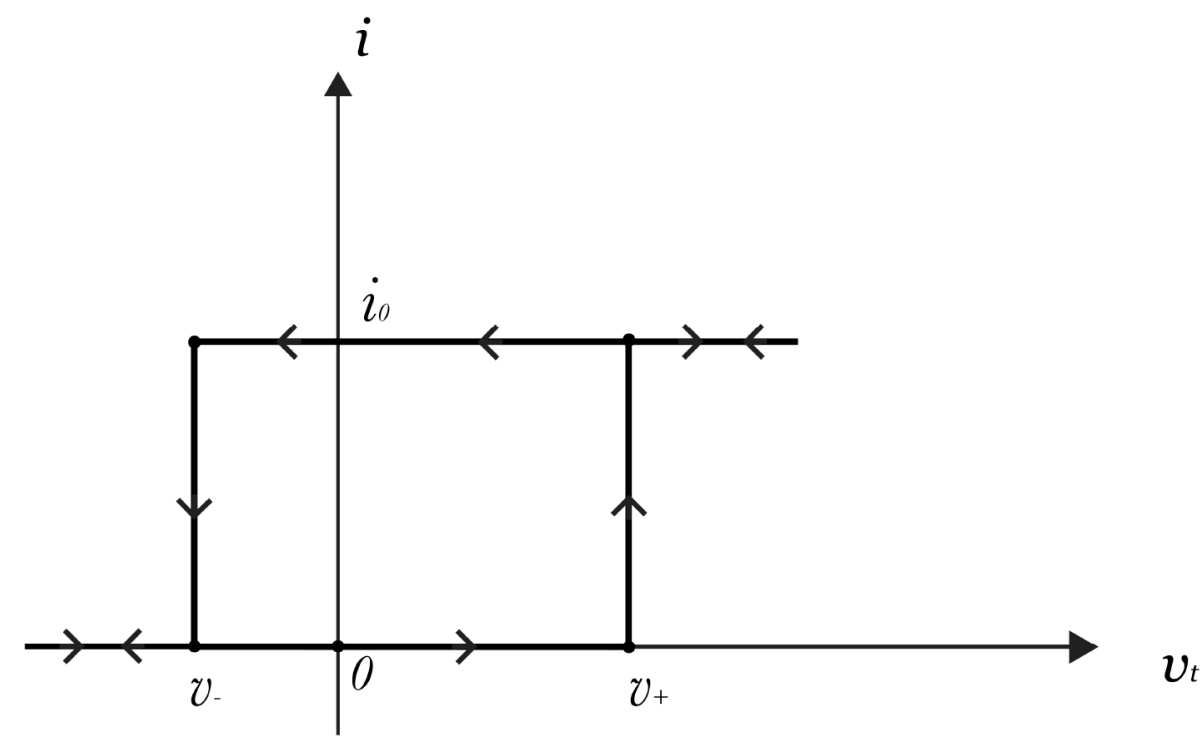

FiguRE 3. Rectangular hysteresis loop.

conformational states. Small noise observed in channel current recordings during the current conduction and its absence (see Fig. 2) can be at least partially attributed to random protein dynamics around their conformational states. Another source of this small randomness in channel current recordings may be the electronic noise of the measuring equipment itself.

Classical kinetic theory was developed to account for random currents through ion channels. It is postulated in this theory that there are certain protein conformational states and Markov random processes are used to describe switching between these states. These switchings are usually illustrated by kinetic diagrams with fixed switching probability rates (called kinetic constants) between conformational states. This underlying Markovian assumption is not consistent with the hysteresis phenomena exhibited by ion channels. The presence of hysteresis implies that ion channels are endowed with memory. Furthermore, very interesting research was performed to test the validity of the Markovian condition for ion channel current recordings. The idea of testing this condition was first suggested by Timmer and Klein in [23]. This idea was further developed by Fulinski and his colleagues in [10]. It was demonstrated in [10] that the Markovian condition is not consistent with experimental data obtained for some potassium channels. It is therefore interesting to develop the theory of random channel currents which is free from the underlying Markovian assumption. One way of doing this is to consider the stochastic channel currents as being produced by random switching of rectangular hysteresis loops. This is illustrated by Figure 3 .

In this figure, the vertical axis corresponds to channel current, while the horizontal axis corresponds to the membrane potential. The membrane potential $v_{t}$ may be considered the clamped potential (in a patch-clamp measurement) or the local potential in the axon in the presence of noise. Hence, in general, this voltage can be considered to be a stochastic process. The choice of the rectangular loop is justified by the fact that in many single channel recordings the random ion currents are binary (as shown in Fig. 2). Furthermore, the switching between closed $(i=0)$ and open $\left(i=i_{0}\right)$ conformations occurs very fast (i.e. on a very short time scale). The latter is reflected in almost vertical lines of transitions between the open and closed states in ion channel current recordings. Switching values $v_{+}$and $v_{-}$depend on conformational states being switched. These states may be changed by gating, and they also may depend on the past history of channel voltages and currents. The channel current at a fixed membrane potential $v$ can be measured by the patch clamp method, and $i_{0}(v)$ data is typically obtained as a result of such measurements.

Mathematically, the hysteresis based random switching between open and closed conformations can be treated as an exit problem for stochastic Markov noise process $v_{t}$ [15]. Indeed, when, as a result of the preceding 


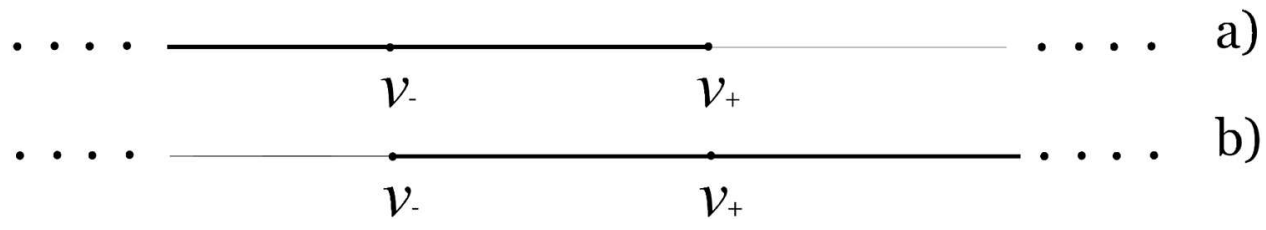

FiguRE 4. Exit intervals of the rectangular hysteresis loop.

a)

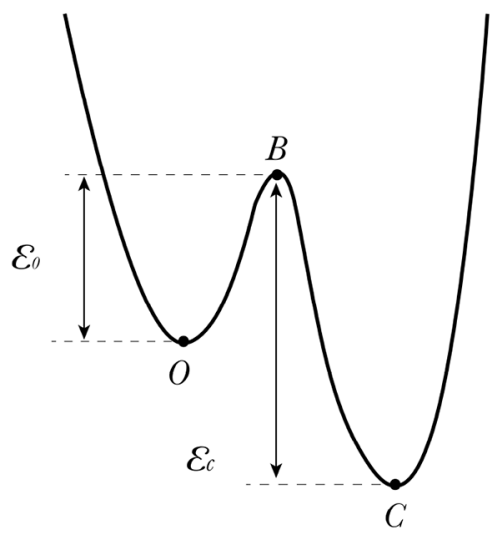

b)

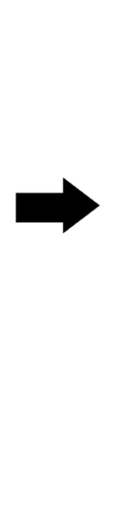

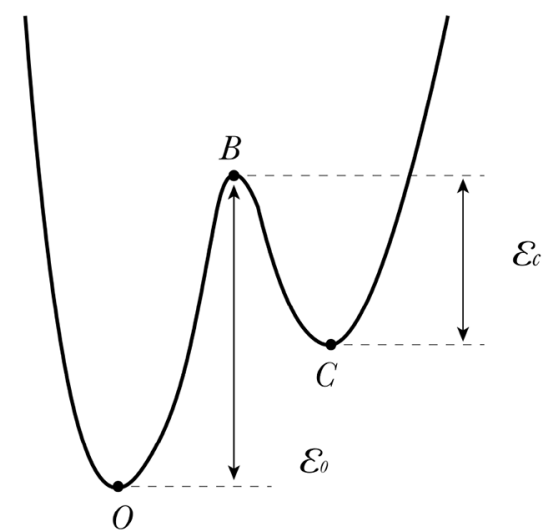

FigURE 5. Energy well structures corresponding to open and closed states of channel proteins.

switching, the random process $v_{t}$ starts from the point $v_{-}$and then exits at the point $v_{+}$on the semi-infinite line shown in Figure 4a, switching from the closed conformations $(i=0)$ to the open conformations $\left(i=i_{0}\right)$ occurs. Similarly, when the random noise $v_{t}$ starts from the point $v_{+}$and then exits at the point $v_{-}$on the semi-infinite line shown in Figure 4b, the switching from the open conformations to the closed conformations occurs. It is apparent that these random switching times are equal to random dwell (duration) times of open and closed conformations. As far as the random process $v_{t}$ is concerned, it may be natural to assume that this is a stationary Gaussian Markov process. It is shown in probability theory that the only process that satisfies these three requirements is the Ornstein-Uhlenbeck process. It must be remarked that the exit problem is one of the classical and well-studied problems in the theory of stochastic processes.

It is worthwhile to point out that the binary current it generated by random switching of a rectangular loop is not Markovian. However, the two-component random process $y_{t}=\left(\begin{array}{l}i_{t} \\ v_{t}\end{array}\right)$ is Markovian. This process is defined on a specific graph representing a rectangular loop in Figure 3. The theory of Markov processes on graphs has been recently developed $[7,8]$. This theory, as well as the theory of exit problems for stochastic processes, can be found in $[9,15]$, respectively. Appendix B presents a summary of stochastic theory on graphs, the basic theoretical framework and key derivations relevant for the stochastic system discussed here.

It is clear from Figure 2 that a single protein channel randomly oscillates between open and closed conformations. These random oscillations occur regardless of whether the ion channel is gated or not. This makes the very definition of open and closed states somewhat ambiguous. It turns out that some clarity in this matter can be introduced by considering energy well structures corresponding to open and closed states of channel proteins, and by analyzing how these energy structures are changed by gating. In the simplest case, these energy structures are schematically shown in Figure 5.

In this figure, energy minima corresponding to open and closed protein conformational configurations are marked by letters $\mathrm{O}$ and $\mathrm{C}$, respectively, while maxima of energy barriers separating adjacent wells are marked by the letter B. It is apparent that the energy structures presented in Figure 5a correspond to closed states. Indeed, for such energy structures, the energy barriers $\varepsilon_{C}$ for closed conformations are appreciably higher than 
the energy barriers $\varepsilon_{O}$ for open conformations. This implies that the random switching from closed conformations to open ones occurs much less frequently than the reverse switching from open conformations to closed ones. This means that on average the channel proteins spend more time around C-conformations than around Oconformations. By gating, the energy structures may be changed to those shown in Figure 5b. It is clear that such energy structures correspond to open states. Indeed, for such energy structures energy barriers $\varepsilon_{C}$ are appreciably smaller than energy barriers $\varepsilon_{O}$. This implies that the random switching from closed conformations to open ones is more probable and, consequently, occur more frequently than the reverse switching from open conformations to closed ones. This means that on average the channel proteins spend more time within the Owells than within the $\mathrm{C}$-wells. The presented discussion clearly reveals that switching from $\mathrm{C}$-wells to $\mathrm{O}$ - wells and from $\mathrm{O}$-wells to $\mathrm{C}$-wells require different switching values of thermal noise energy to overcome different energy barriers. This is properly reflected in the different switching values $v_{+}$and $v_{-}$of the hysteresis loop in Figure 3 .

As discussed before, ion channels are formed by proteins. The remarkable fact is that the proteins are unstable elements. On average, they only last for a couple of days, [4, 18], and then they are replaced by newly synthesized proteins. Thus, there exists a continuous process of protein synthesis and protein degradation. This leads to an unresolved and puzzling issue of how the integrity of neural systems and their memories remain mostly intact in the face of this volatility and fluidity.

\section{Action potentials}

The brain and the entire nervous system function by means of generation and propagation of action potentials. These potentials are short-lasting (in time) spikes of voltages, which are generated by ion channels in neurons. This implies that the action potentials are ionic in nature. It is the established fact that biological fluids inside and outside of neurons contain large numbers of various ions of different concentrations. In particular, the concentrations of sodium $\left(\mathrm{Na}^{+}\right)$, chloride $\left(\mathrm{Cl}^{-}\right)$, and calcium $\left(\mathrm{Ca}^{2+}\right)$ ions are appreciably higher outside the neurons than inside. Whereas, the concentration of potassium $\left(\mathrm{K}^{+}\right)$ions is much higher inside rather than outside the neurons. Under unperturbed conditions, these ion concentrations result in voltages across neuron bilipid membranes, which are typically between $60 \mathrm{mV}$ and $70 \mathrm{mV}$. If the potential of the exterior side of the membrane is chosen to be equal to zero, then the potential of the interior membrane side is between $-70 \mathrm{mV}$ and $-60 \mathrm{mV}$. This potential is called the resting potential. This potential is maintained by continuous ion flow across membrane realized by ionic pumps. In this sense, the resting potential is not an equilibrium state potential. Maintaining this potential is the major energy expenditure in the nervous system.

When a neuron soma is electrically perturbed by signals coming from its dendrites or by an external stimulus, action potentials may be generated at an axon initial segment. This segment has a very high concentration of sodium and potassium ion channels, and this makes this part of the neuron very sensitive to electrical perturbations of its resting potential. In other words, the axon initial segment is a "trigger zone" that is the most excitable part of the neuron. For the action potential to be generated, the perturbed membrane potential must exceed some threshold value, which is called the threshold potential. This threshold potential is about $15 \mathrm{mV}$ higher than the resting potential. As soon as the threshold potential is exceeded, a brief voltage spike is generated and starts to propagate along the axon. These spikes are peaked between $+20 \mathrm{mV}$ and $+40 \mathrm{mV}$. One typical shape of the action potential generated at the axon initial segment is schematically shown in Figure 6.

It is evident from the above figure that there are three distinct phases in the time evolution of the action potential. They are the rising phase, the falling phase, and the undershoot phase. During the rising phase the membrane potential monotonically increases and changes its sign from negative to positive. That is why this phase is called the depolarization phase. The time duration of this phase is about $1 \mathrm{~ms}$. During the falling phase, the membrane potential monotonically decreases and returns to its negative value. For this reason, this phase is called repolarization phase. Its time duration is about $2 \mathrm{~ms}$. During the depolarization phase, the membrane potential decreases below its resting value. For this reason, this phase is followed by the subsequent monotonic increase of membrane potential back to its resting value. This phase of restoration of the membrane potential is called hyperpolarization phase. Its time duration is about $5 \mathrm{~ms}$. It is interesting to note that this hyper 


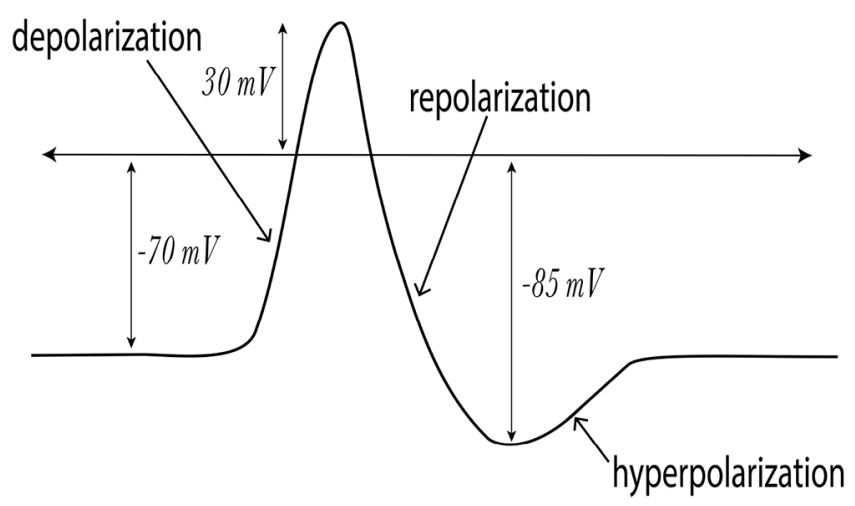

FIGURE 6. Schematic of a typical action potential.

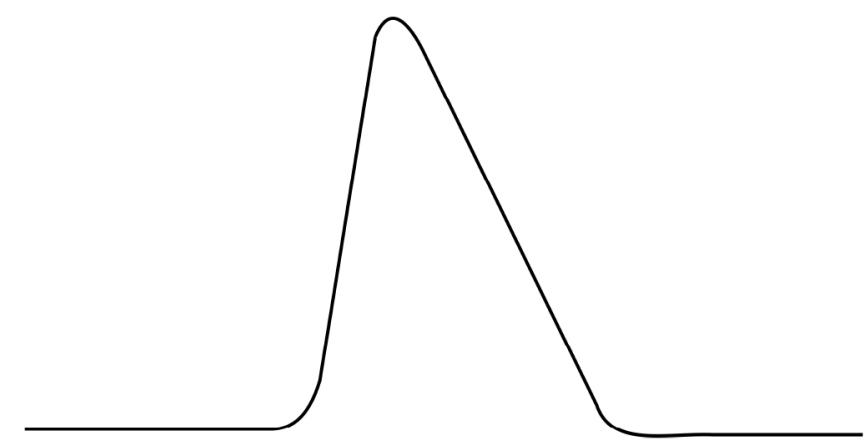

FIgURE 7. Typical shape of the action potential at the node of Ranvier.

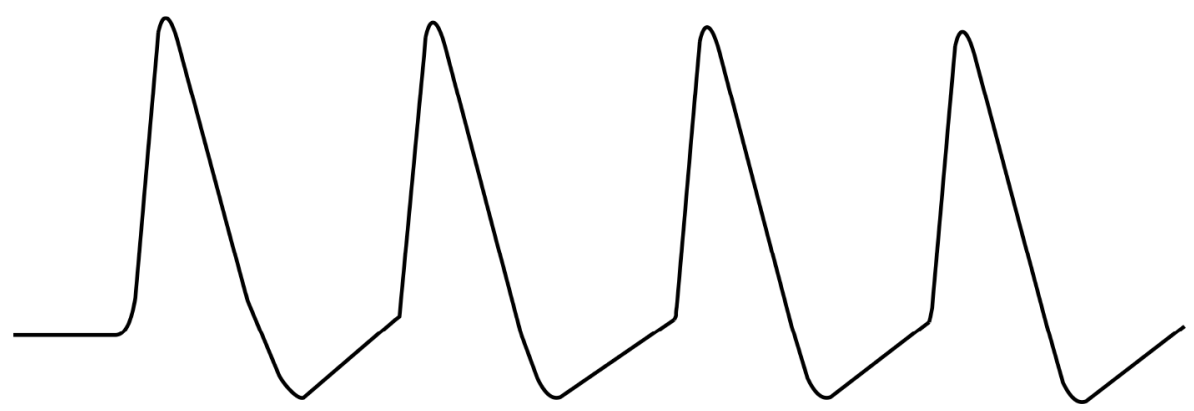

FiguRE 8. Train of action potentials at the node of Ranvier.

polarization phase is absent for action potentials generated at Ranvier nodes of myelinated axons. A typical shape of the action potential at the node of Ranvier is schematically shown in Figure 7.

Typically, not a single action potential, but rather a sequence (a train) of action potentials is generated, and these potentials propagate along the axon. Remarkably, these action potentials are identical (i.e. stereotyped) in shape. Schematically, a train of such action potentials is shown in Figure 8.

It is evident from this figure that the voltage spikes (action potentials) have a certain separation in time, which is called the refractory period. This period can be subdivided into two distinct parts: an absolute refractory period during which no generation of action potential is possible, then followed by a relative refractory period when a stronger-than-usual stimulus is required to generate the next action potential. The physical nature of 
the relative refractory period is quite apparent. Indeed, if the membrane potential is below the resting potential, then a stronger stimulus is needed in order to exceed the threshold potential. On the other hand, the absolute refractory period is related to the unique feature of the sodium channel, related to their inactivated state, which will be discussed later.

Next, we consider the physical mechanism of the generation of action potentials. At the very foundation of this mechanism are the properties of voltage-gated sodium and potassium channels. At the beginning of the depolarization phase when the membrane potential at a certain location exceeds the threshold potential, some voltage-gated channels become open at this location. This results in the influx of positively charged sodium ions into a neuron which leads to the further increase of the membrane potential at this and adjacent locations. Consequently, new voltage-gated sodium channels get open, which brings about an additional influx of positively charged sodium ions. This further increases the local membrane potential and results again in activation of additional sodium channels. Thus, it is clear that there exists a strong positive feedback at work here, which results in a steep local increase in membrane potential. This membrane depolarization is aborted by inactivation of sodium channels resulting in some peak value of action potential. At this stage, voltage-gated potassium channels get activated, leading to the outflux of positively charged potassium ions from the neuron. This brings about the repolarization phase of membrane potential. When voltage-gated channels finally close, the resting potential is re-established during the hyperpolarization phase. There is no consensus in the literature concerning the physical mechanism of the restoration of the resting potential during the hyperpolarization phase. The most frequently expressed point of view is that this restoration occurs due to the pump (and leak) channels.

It is apparent from the above description of the action potential generation (as well as from Fig. 8) that as soon as the threshold membrane voltage is exceeded, the action potentials of identical (stereotyped) shape are produced. If the perturbed membrane potential is below its threshold value, then no action potential is generated. This is the essence of the all-or-none principle. The origin of this principle is due to the positive feedback that drives the generation of action potentials. As a result of this feedback, currents produced by the opening of sodium voltage-gated channels are much larger than stimulating currents. Consequently, if the threshold voltage is exceeded, then amplitudes and shapes of action potentials are determined by the properties of membrane channels rather than by the strength of the initial stimulus. For this reason, these shapes are stereotyped.

Next, we shall discuss two important and difficult issues related to the generation of action potentials. The first issue is related to deterministic stereotyped shapes of action potentials produced by ionic channels despite the fact that the current conduction of individual channels is stochastic in nature (as discussed in the previous section). It is intuitively clear that some averaging over large number of ionic channels is involved in the deterministic representations of the action potentials. In this sense, the deterministic action potentials can be viewed as macroscopic potentials while random characteristics of individual ion channels can be regarded as microscopic ones. The issue of how purely deterministic action potentials emerge from stochastic actions of individual channels as a result of averaging is very interesting and a difficult one. In our discussion, this matter will be left unattended.

The second issue is related to the states of voltage-gated sodium channels. To summarize, there are three states of voltage-gated sodium channels involved in the formation of action potentials. They are the deactivated (closed) state, activated (open) state, and inactivated state. During the action potential generation, voltage-gated sodium channels go through the following transition cycle: deactivated $\rightarrow$ activated $\rightarrow$ inactivated $\rightarrow$ deactivated. The physical mechanism of the deactivated $\rightarrow$ activated transition is more or less clear. Namely, the applied voltage across the neuron membrane at the location of sodium channels modifies the energy well structures of open and closed conformational configuration of channel proteins by making the open conformation more probable. In contrast, the physical mechanism of the activated $\rightarrow$ inactivated transition is much less understood. There are several factors to consider. This transition is usually associated with the existence of a special "inactivated" gate at the intracellular side of the ion channel, and efforts have been made to interpret this gate in terms of channel protein structure, as described in the work of W. A. Catterall [1]. However, there exists a fundamental difference between "deactivated $\rightarrow$ activated" and "activated $\rightarrow$ inactivated" transitions. The first transition occurs with no current through a sodium channel, while the second transition takes place when the ion flow through the 


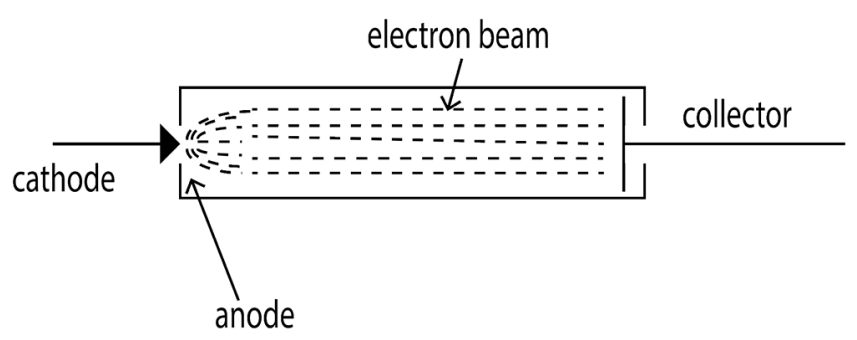

Figure 9. Schematic of an emission diode.

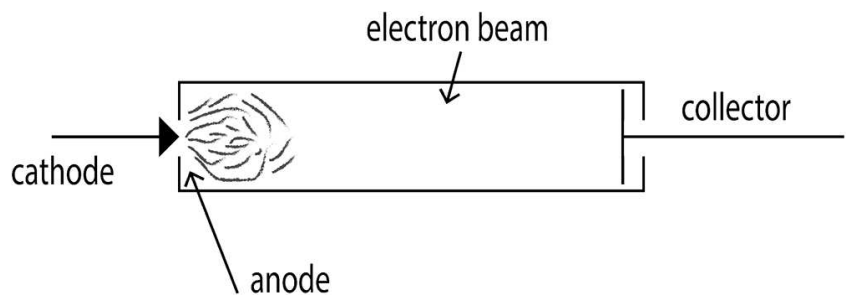

FiguRE 10. Schematic of a virtual cathode formed by an electron cloud in close proximity of the anode.

sodium channel is quite large, even at its peak. Since the switching to the inactivated state occurs during ion current flow, this suggests that the transition from the activated to the inactivated state cannot be explained by membrane potential control alone. It is conceivable that the ion flow itself may be the cause of the transition to the inactivated state. With this in mind, we propose two plausible ion current controlled mechanisms that play a key role during the activated state and, as a result, cause the transition to the inactivated state.

Indeed, a similar phenomenon has been observed for electron beams in emission diodes [17]. Such a diode is schematically shown in Figure 9. Here, the emitted electrons are accelerated by the voltage applied between the cathode and the anode and propagate through the anode hole into the drift region between the anode and the collector. Such an electron beam has unique nonlinear properties. Namely, if the electron beam current $I$ is below some critical value called the limiting current, $I_{L}$, then the electrons propagate toward the collector, and the full electron beam current is usually measured at the collector site. However, if the electron beam current $I$ exceeds $I_{L}$, then an electron cloud (cluster) called a virtual cathode is formed in the close proximity to the anode as pictured in Figure 10. In this situation, almost all current above the limiting value $I_{L}$ is diverted to the anode. This phenomenon occurs naturally due the very strong Coulomb repulsion between similarly charged electrons.

Remarkably, the described physical phenomenon exhibits hysteresis between the injected electron beam current $I$ and reflected electron current $I_{R}$. This hysteresis is schematically shown in Figure 11 .

According to this figure, if the injected electron beam current $I$ is monotonically increased, then there is no reflected current until $I$ exceeds the threshold value $I_{L}$. After $I$ exceeds $I_{L}$, the virtual cathode is formed and most of the injected current $I$ is reflected. In the case of the subsequent monotonic decrease of $I$, this reflection persists until $I$ is reduced below the so-called space charge limiting current $I_{L S}$ which may be substantially smaller than $I_{L}$. Then, the virtual cathode disappears and the reflection seized to exist. The physical mechanism of the described irreversibility and hysteresis is based on the fact that in order to form the virtual cathode, an injected current $I$ above the limiting current ${ }_{L}$ is needed, whereas in order to maintain the virtual cathode, injection currents only above the space-charge-limited value is sufficient. In other words, when $I$ exceeds $I_{L}$, the emission diode is switched into "inactivated" state which persists until the injection current is reduced below $I_{S C}$. 


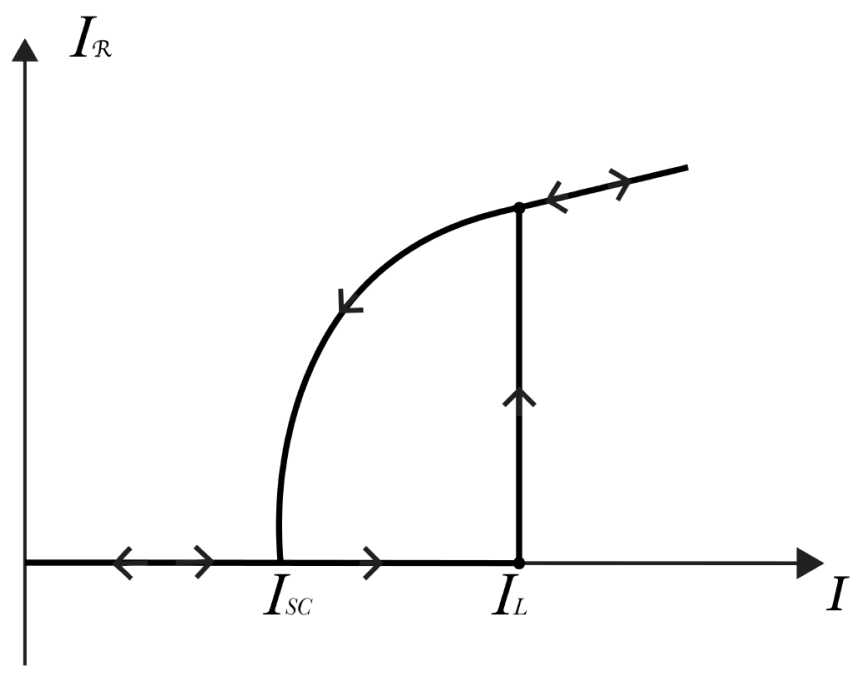

FigURE 11. Electron beam hysteresis curve.

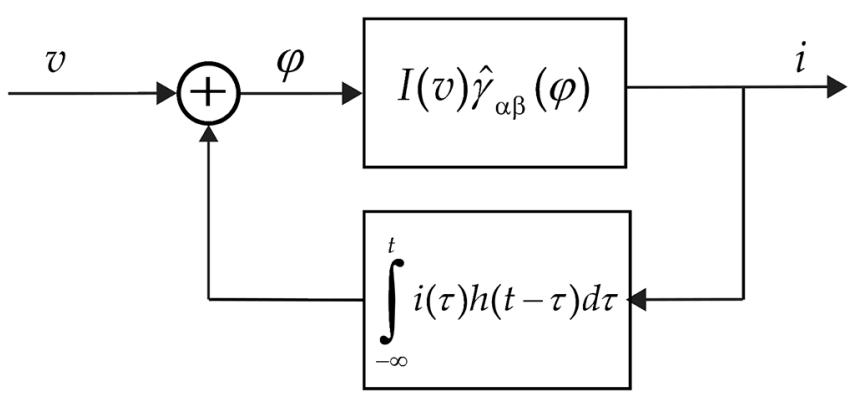

Figure 12. Sodium ion channel feedback loop.

It is conceivable that a similar phenomenon may occur in voltage-gated sodium channels. Namely, when the ions current through the sodium channel approaches its peak, the strong repulsive Coulomb (electrostatic) interaction between ions may lead to the formation of the ion accumulations (ion clusters) within the channels, which may result in sudden decrease and in eventual stoppage of ion currents through the channels. This is a plausible mechanism for the activated $\rightarrow$ inactivated transition. These ion clusters may persist over time during the repolarization phase resulting in the persistence of the inactivated state. The presented idea of formation and persistence of the inactivated state merits further investigation along with the study of other affects which may be caused by ion flows through the channels.

Since the activated $\rightarrow$ inactivated sodium ion channel transition occurs during current flow, this suggests that switching between these two metastable states may be due to Coulombic interaction of channel ions in addition to the membrane potential. In order to pursue this line of reasoning further, one may consider an ion current feedback mechanism. Let us consider a sodium ion channel feedback model as shown in Figure 12.

According to the model, the ion channel is controlled by the membrane potential $v$ and feedback of the time integral of the channel current $i$. Here, $I(v)$ represents the ion current when the channel is open, and the membrane potential is held at a constant potential $v$ subsequent to a voltage step from the deactivated state (as measured with standard patch clamp techniques). To be consistent with standard ion channel measurements, by convention, $I(v)$ is negative in value. The binary hysteresis operator, as shown in Figure 13, depicts the status of the channel where output 0 corresponds to a closed channel and output 1 corresponds to an open channel. 


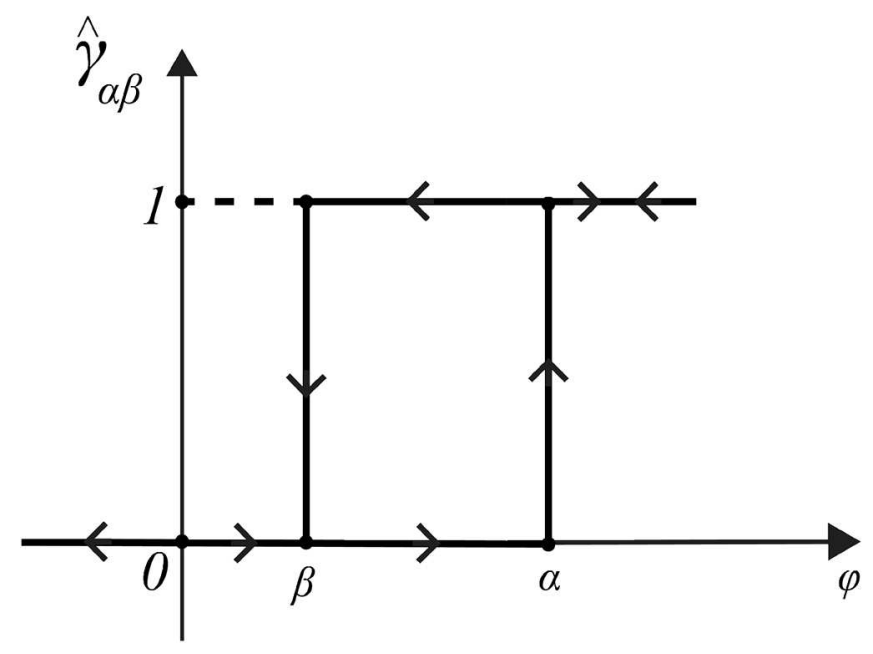

FIGURE 13. Sodium ion channel hysteresis.

Here, $\alpha$ and $\beta$ denote the values of the membrane potential to switch the channel between the deactivated $\rightarrow$ activated and the activated $\rightarrow$ deactivated states, respectively, in the absence of ion currents.

The feedback operator represents some accumulated level of channel ion current charge and it is modeled as a time invariant linear system. It is clear that some negative feedback is needed to close the channel due to current flow. Since the channel current i is negative (by convention), the impulse response $h(t)$ should be a positive function.

Let us examine this mechanism more carefully. Consider the channel in the deactivated state and the membrane potential $v<\alpha$, where $v$ in practice is a large negative voltage less than the resting potential. Therefore, the channel is closed and the channel current is zero. Next, the voltage is stepped up to some level above the rest potential, i.e., $v>\alpha$, and is maintained at that value until the next measurement cycle. Now, since $v>\alpha$, the rectangular loop switches to 1 , and there is sodium ion current $I(v)$. Without feedback and in the absence of noise, this current will flow indefinitely, and the channel will remain in the activated state. However, patch clamp measurements show that this does not occur, and the current is turned off after a few milliseconds of current flow. It is worth noting here that, due to the stochastic nature of the system, the ion channel current may fluctuate between $I(v)$ and 0 , corresponding to the 1 and 0 states of the rectangular hysteresis loop, respectively. Such binary step like fluctuations, commonly observed in patch clamp measurements and schematically depicted in Figure 2, correspond to the transitions of the rectangular hysteresis loop as the channel stochastically opens and closes until eventually reaching the inactivated state.

Now, let us consider the simplest choice for the feedback function: a scaled unit step function, $h(t)=K u(t)$, where $K>0$ (Volts/Coulomb). It is clear that such a choice represents a scaled value of the cumulative ion current charge flow during the activation of the ion channel. Since, by convention, the influx sodium ion current is negative, the feedback loop represents a negative feedback, $K I(v) T<0$, where $T$ is the total (random) time the channel stays open. Therefore, this negative feedback produces an effective input of $(v+K I(v) T)$ to the rectangular hysteresis loop. Since the membrane potential $v>\alpha$ in this illustration, i.e, large enough to result in the activated state, it is clear that this effective input may result in $(v+K I(v) T)<\beta$. This would clearly results in closing the channel, which the membrane potential $v>\alpha$ only cannot do in the absence of any other fluctuations.

Clearly, one may consider a wide variety of ion current feedback models, different patch clamp membrane potential protocols and, more importantly, operation of these feedback mechanism under action potentials. 


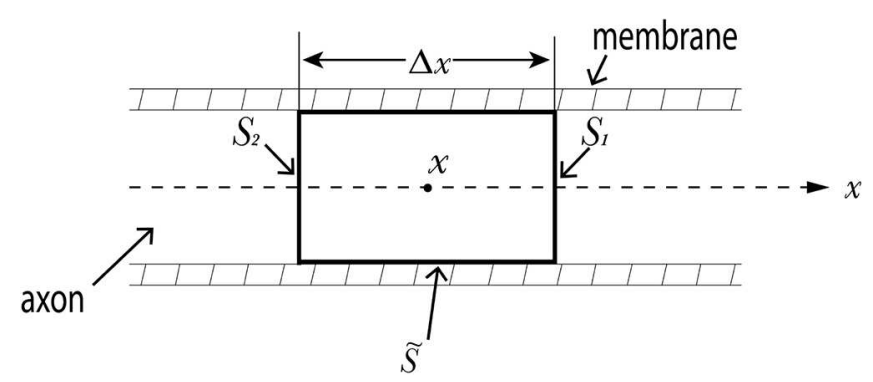

Figure 14. Axon cross section schematic.

However, the detailed study of this feedback mechanism is left to future work, and will not be pursued further in this paper.

Next, we proceed to the derivation of the deterministic nonlinear diffusion equation for generation and propagation of action potentials. To this end, we consider a very short (in a macroscopic sense) cylindrical region of unmyelinated axon shown in Figure 14. Here $x$ is a central point of this cylinder, while $\Delta x$ is its "macroscopic" length. The latter means that the number of microscopic membrane channel along cylindrical side surface $\tilde{S}$ is very large.

The subsequent derivation is based on the principle of continuity of electric current, which states that the following mathematical relation is valid for any closed surface $S$ :

$$
\oiint_{S} \mathbf{J} \cdot \mathrm{d} \mathbf{s}=0
$$

where $\mathbf{J}$ is an electric current density, while the direction of ds coincides with the direction of outward normal to $S$.

By applying the last formula to the cylindrical surfaces shown (in bold) in Figure 14, we find:

$$
\iint_{\tilde{S}} \mathbf{J} \cdot \mathrm{d} \mathbf{s}+\iint_{S_{1}} \mathbf{J}_{a} \cdot \mathrm{d} \mathbf{s}+\iint_{S_{2}} \mathbf{J}_{a} \cdot \mathrm{d} \mathbf{s}=0
$$

where the notation $\mathbf{J}_{a}$ is used for the axial (along $x$ ) component of electric current density.

The first integral in the left-hand side of formula (3.2) can be represented as follows:

$$
\iint_{\tilde{S}} \mathbf{J} \cdot \mathrm{d} \mathbf{s}=2 \pi r \Delta x J_{\text {sides }}
$$

where $r$ is the axon radius, while $J_{\text {sides }}$ is the average (over $\tilde{S}$ ) value of current density normal to the side surface $\tilde{S}$.

The sum of the last two integrals in the left-hand side of formula (3.2) can be written as the difference of the two axial currents $I_{a}\left(x+\frac{\Delta x}{2}\right)$ and $I_{a}\left(x-\frac{\Delta x}{2}\right)$ leaving and entering, respectively, the cylindrical axon region under consideration:

$$
\iint_{S_{1}} \mathbf{J}_{a} \cdot \mathrm{d} \mathbf{s}+\iint_{S_{2}} \mathbf{J}_{a} \cdot \mathrm{d} \mathbf{s}=I_{a}\left(x+\frac{\Delta x}{2}\right)-I_{a}\left(x-\frac{\Delta x}{2}\right)
$$


By substituting the last two formulas into the equation (3.2), we find:

$$
2 \pi r J_{\text {sides }}+\frac{I_{a}\left(x+\frac{\Delta x}{2}\right)-I_{a}\left(x-\frac{\Delta x}{2}\right)}{\Delta x}=0 .
$$

Since $\Delta x$ is macroscopically small, the last term in the left-hand side of formula (3.5) can be replaced by the derivative, which leads to the following equation:

$$
2 \pi r J_{\text {sides }}+\frac{\partial I_{a}}{\partial x}=0
$$

The current $J_{\text {sides }}$ can be represented as a sum of average displacement (capacitive) current $J_{\text {dis }}$ through $\tilde{S}$ and the average ion current $J_{c h}(\varphi)$ through membrane channels along $\tilde{S}$ :

$$
J_{\text {sides }}=J_{d i s}+J_{c h}(\varphi)
$$

The ion current through $\tilde{S}$ depends on (average) potential $\varphi$ on $\tilde{S}$, which is reflected in its notation. On the other hand, the average displacement current can be written as follows:

$$
J_{d i s}=C \frac{\partial \varphi}{\partial t}
$$

where $C$ is the per unit surface membrane capacitance.

In the case when an axial diffusion current of ions within the axon is a small effect in comparison with the ion drift current, then the axial current $I_{a}$ is only of resistive nature and can be represented by formula:

$$
I_{a}=-\frac{1}{R} \frac{\partial \varphi}{\partial x}
$$

where $R$ is the per unit length resistance of the axon.

By substituting the last three formula into (3.6) we end up with the following nonlinear diffusion equation:

$$
b \frac{\partial \varphi}{\partial t}-\frac{\partial^{2} \varphi}{\partial x^{2}}+f(\varphi)=0
$$

where

$$
b=2 \pi r R C
$$

and

$$
f(\varphi)=2 \pi r R J_{c h}(\varphi)
$$

The derived diffusion equation (3.10) is nonlinear because the term $f(\varphi)$ which is proportional to average channel current density is a nonlinear function of $\varphi$. The nonlinear diffusion equation (3.10) was (and still is) the foundation of the Hodgkin Huxley theory of the action potential generation and propagation. It is worthwhile to mention that Hodgkin and Huxley developed their theory when the existence of ionic channels and their individual stochastic nature were not known.

Hodgkin and Huxley realized the importance of sodium and potassium ion currents through a neuron membrane for the generation and propagation of action potentials. They introduced these currents (along with small 
leakage currents) into a diffusion equation as nonlinear functions of some gating variables (a term not used by Hodgkin and Huxley but later adopted in the neural science literature). Furthermore, they proposed separate first order ordinary differential equations with respect to time for these variables with coefficients being nonlinear functions of potential $\varphi$. Finally, Hodgkin and Huxley used these equations to numerically study the finite speed propagation of action potentials for unmyelinated axons and compare their computations with experimental results obtained for the giant axon of the squid.

Below, we describe another inverse problem approach which reveals the hysteretic nature of macroscopic sodium and potassium ion currents. The central idea of this approach is to treat the functions $f(\varphi)$ in the nonlinear diffusion equation (3.10) as unknown and determine it from such experimental data as the measured deterministic shape of the action potential and its finite speed of propagation. Such a speed does not exist for linear diffusion equations, but it is quite possible for nonlinear diffusion equations.

To start the discussion, we assume that the solution of equation (3.10) has the form:

$$
\varphi(x, t)=\varphi(v t-x)
$$

where function $\varphi$ and speed $v$ are known (they have been measured). We want to determine the function $f(\varphi)$ in equation (3.10) from its solution (3.13). To do this, we introduce the variable:

$$
\lambda=v t-x
$$

and, according to equation (3.13), we treat the electric potential as a function of this variable:

$$
\varphi(x, t)=\varphi(\lambda)
$$

It is apparent that

$$
\frac{\partial \varphi}{\partial t}=v \varphi^{\prime}(\lambda)
$$

and

$$
\frac{\partial^{2} \varphi}{\partial x^{2}}=\varphi^{\prime \prime}(\lambda)
$$

By substituting the last two formulas into the nonlinear differential equation (3.10), we obtain:

$$
v b \varphi^{\prime}(\lambda)-\varphi^{\prime \prime}(\lambda)+f[\varphi(\lambda)]=0
$$

which leads to

$$
f[\varphi(\lambda)]=-v b \varphi^{\prime}(\lambda)+\varphi^{\prime \prime}(\lambda)
$$

The last equation can be used to determine the function $f(\varphi)$. We shall demonstrate this for the simple case of the triangular shaped action potential shown in Figure 15.

The triangular shape can be viewed as a reasonable approximation of the actual shape of the action potential. It is also clear that the linear upstroke of triangular shape is produced by sodium ion currents, while the linear potential reversal is due to the potassium ion currents. For this reason, function $f(\varphi)$ can be split into the sum of two functions $f_{\mathrm{Na}^{+}}(\varphi)$ and $f_{K^{+}}(\varphi)$ corresponding to sodium and potassium currents, respectively:

$$
f_{N a^{+}}(\varphi)+f_{K^{+}}(\varphi)=f(\varphi)
$$




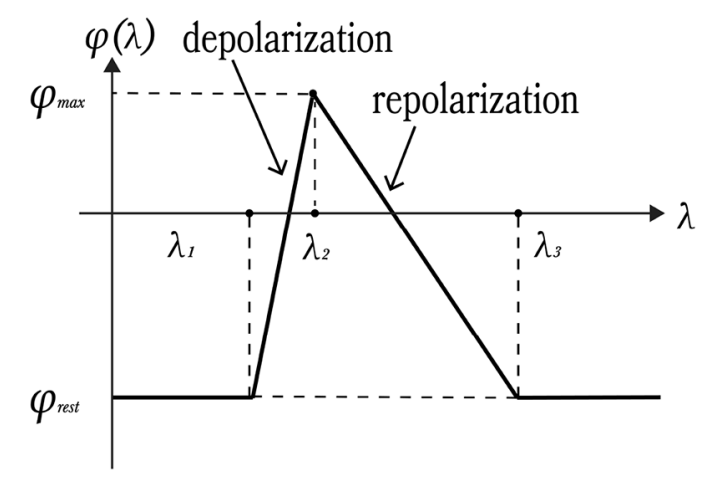

FiguRE 15. Triangular shaped action potential.

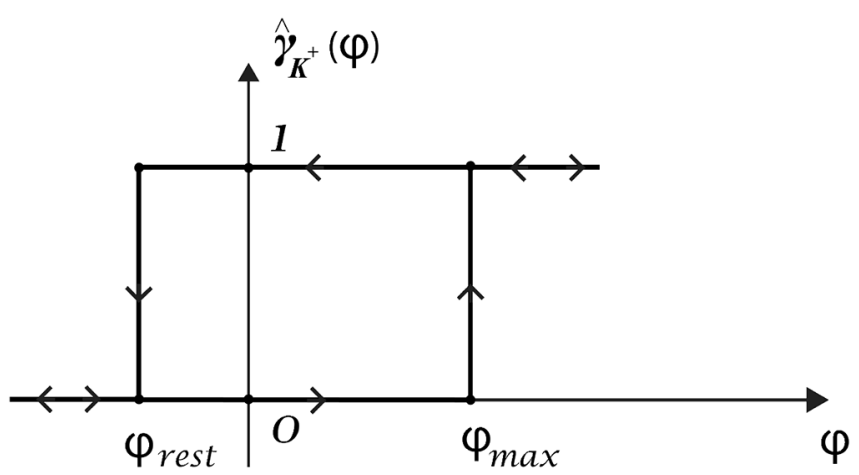

FiguRE 16. Potassium ion channel hysteresis.

Now from Figure 15 and formula (3.19) we find

$$
f_{N a^{+}}(\varphi)= \begin{cases}-v b \frac{\varphi_{\max }-\varphi_{\text {rest }}}{\lambda_{2}-\lambda_{1}} & , \text { if } \lambda_{1}<\lambda<\lambda_{2} \\ 0 & , \text { otherwise }\end{cases}
$$

Similarly, from Figure 15 and equation (3.19), we derive

$$
f_{K^{+}}(\varphi)= \begin{cases}v b \frac{\varphi_{\max }-\varphi_{\mathrm{rest}}}{\lambda_{3}-\lambda_{2}} & , \text { if } \lambda_{2}<\lambda<\lambda_{3} \\ 0 & , \text { otherwise }\end{cases}
$$

As expected, the term $f_{\mathrm{Na}^{+}}(\varphi)$, corresponding to sodium current is nonpositive because of the influx of $\mathrm{Na}^{+}$ ions, whereas the term $f_{K^{+}}(\varphi)$ is nonnegative because of outward flow of $\mathrm{K}^{+}$ions.

It turns out that sodium and potassium ion current terms can be represented in terms of rectangular hysteresis loops. To demonstrate this, we start with the potassium ion current and introduce the hysteresis loop shown in Figure 16.

From formula (3.22) and Figure 16, it is clear that $f_{K^{+}}(\varphi)$ can be expressed as follows:

$$
f_{K^{+}}(\varphi)=v b \frac{\varphi_{\max }-\varphi_{\text {rest }}}{\lambda_{3}-\lambda_{2}} \hat{\gamma}_{K^{+}}(\varphi) .
$$




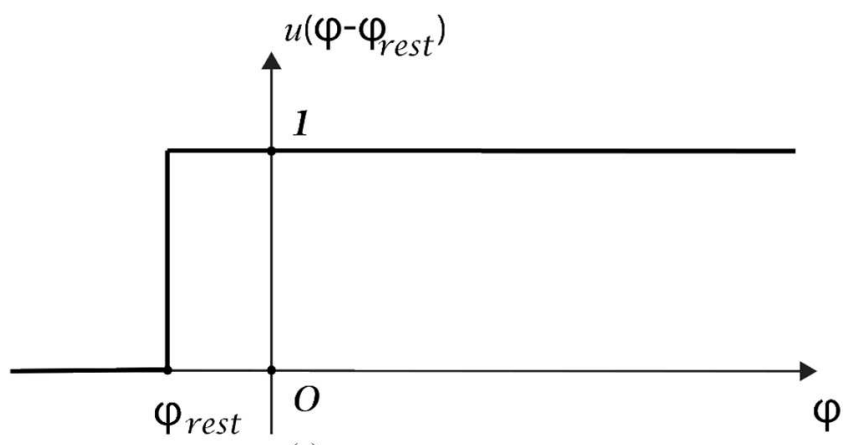

(a)

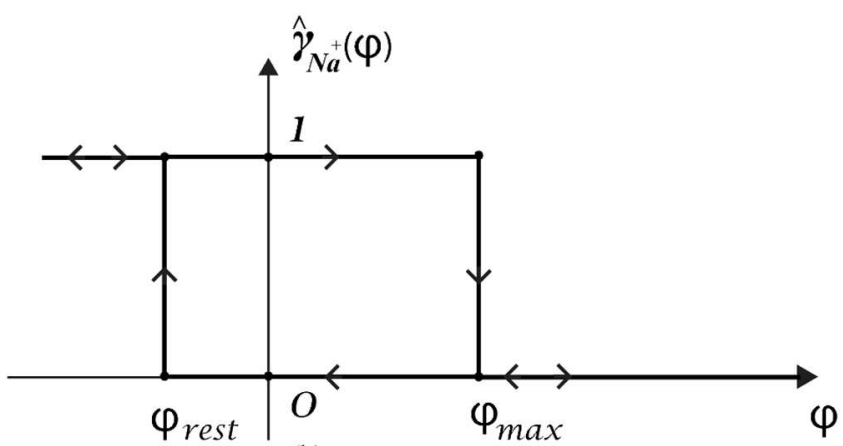

(b)

FiguRE 17. Sodium ion channel hysteresis.

It is clear that the use of one two-state rectangular hysteresis operator is sufficient to represent potassium current, and this is consistent with the fact that the underlying potassium ion channels have only two states, i.e., deactivated and activated.

The sodium current given in (3.21) can also be expressed similar to formula (3.23). However, it requires one key modification because in order to realize formula (3.21) one needs three states. Indeed, unlike the potassium current, the sodium current is a result of the collective action of ion channels that have three states, i.e., deactivated, activated and inactivated. With this in mind, it is clear that $f_{\mathrm{Na}^{+}}(\varphi)$ can be expressed as follows:

$$
f_{N a^{+}}(\varphi)=-v b \frac{\varphi_{\max }-\varphi_{\text {rest }}}{\lambda_{2}-\lambda_{1}} u\left(\varphi-\varphi_{\text {rest }}\right) \hat{\gamma}_{N a^{+}}(\varphi)
$$

Here, $u$ denotes the unit step function which is shown in Figure 17a, and $\hat{\gamma}_{\mathrm{Na}^{+}}(\varphi)$ is the hysteresis loop shown in Figure 17b. According to this formula and Figure 17, we can conclude that the sodium current term $f_{\mathrm{Na}^{+}}(\varphi)$ is equal to zero if $\varphi<\varphi_{\text {rest }}$, then it jumps to its negative value given by the formula (3.21) as $\varphi$ reaches $\varphi_{\text {rest }}$, it remains at this negative value as the potential $\varphi$ is monotonically increased between $\varphi_{\text {rest }}$ and $\varphi_{\max }$. Finally, this current term jumps back to zero as $\varphi$ reaches $\varphi_{\max }$ and it equals to zero as the potential $\varphi$ is decreased from $\varphi_{\max }$ to $\varphi_{\text {rest }}$, and it remains zero thereafter.

The analysis for the action potential approximation depicted in Figure 15 can be generalized to model the hyperpolarization phase, as well. Consider the addition of the hyperpolarization phase, as shown in Figure 18. In this modification, the decrease of the action potential due to the influx of potassium ions continues until the membrane potential, $\varphi$, decreases monotonically to a minimum potential $\varphi_{\min }$ below the rest potential $\varphi_{\text {rest }}$. When $\varphi$ reaches $\varphi_{\min }$, the potassium influx stops, and the membrane potential starts to rise as the equilibrium rest potential, $\varphi_{\text {rest }}$, is eventually restored. For this modification, the potassium ion channel hysteresis shown 


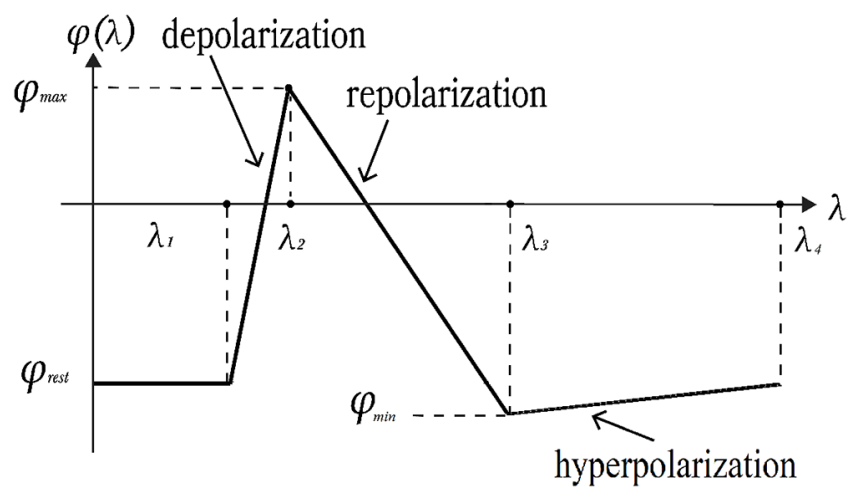

FiguRE 18. Action potential with hyperpolarization.

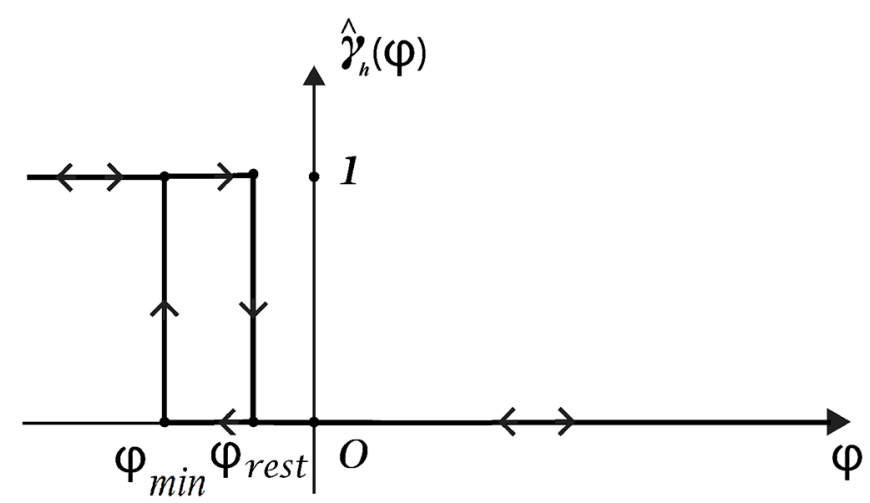

FiguRE 19. Hyperpolarization hysteresis loop.

in Figure 16 needs to be modified by replacing the downward switching threshold $\varphi_{\text {rest }}$ with the new minimum potential $\varphi_{\min }$.

Accordingly, the function $f(\varphi)$ can now be split into the sum of three functions $f_{N a^{+}}(\varphi), f_{K^{+}}(\varphi)$ and $f_{h}(\varphi)$, as follows:

$$
f(\varphi)=f_{N a^{+}}(\varphi)+f_{K^{+}}(\varphi)+f_{h}(\varphi)
$$

where $f_{h}(\varphi)$ represents the process during hyperpolarization that restores the potential to $\varphi_{\text {rest }}$. Following similar lines of reasoning, the analysis presented in expressions (3.13)-(3.24) can easily be updated. Expression (3.23) for the potassium ion current is modified to be:

$$
f_{K^{+}}(\varphi)=v b \frac{\varphi_{\max }-\varphi_{\min }}{\lambda_{3}-\lambda_{2}} \hat{\gamma}_{K^{+}}(\varphi)
$$

noting that in this expression $\hat{\gamma}_{K^{+}}(\varphi)$ is the potassium channel hysteresis with the (modified) switching down value $\varphi_{\min }$. Similarly, the term $f_{h}(\varphi)$, representing the restoration of the rest potential during the hyperpolarization phase, is given as follows:

$$
f_{h}(\varphi)=-v b \frac{\varphi_{\mathrm{rest}}-\varphi_{\min }}{\lambda_{4}-\lambda_{3}} \hat{\gamma}_{h}(\varphi)
$$

where $\hat{\gamma}_{h}(\varphi)$ is the hysteresis loop shown in Figure 19. 


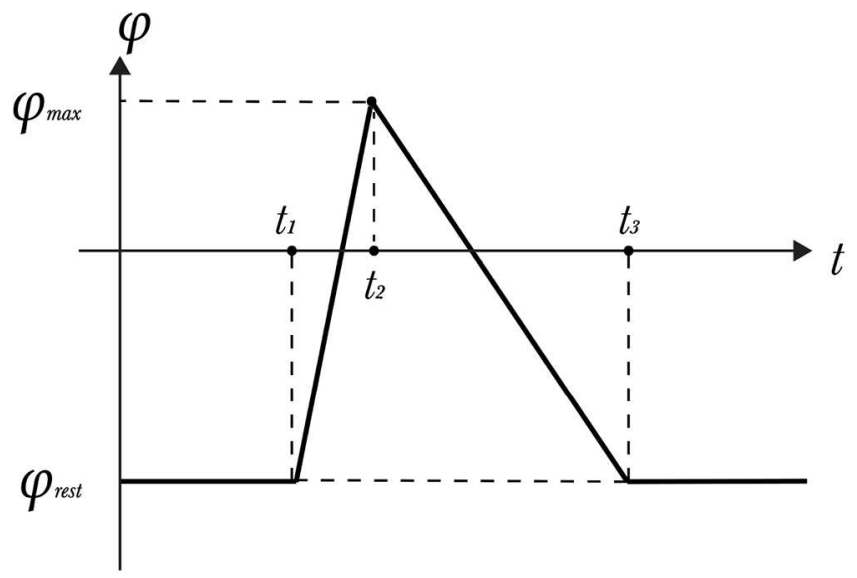

FiguRE 20. Triangular shaped action potential.

Now, we consider regeneration of action potentials at the nodes of Ranvier of myelinated axons. These action potentials do not have the hyperpolarization phase (see Fig. 7) and, for this reason, they can be reasonably well approximated by a triangular shape shown below in Figure 20.

As discussed earlier, nodes of Ranvier are separated by myelinated segments (see Fig. A.1), and there are no ion channels embedded in axon membranes along these segments. Whereas, there are high concentrations of ion channels at nodes of Ranvier. This implies that the electric potential along the myelinated axon membrane is described by two separate equations, which are particular cases of the diffusion equation (3.10). Namely, the electric potential $\varphi$ along myelinated inter-node segments satisfies the linear diffusion equation:

$$
b \frac{\partial \varphi}{\partial t}-\frac{\partial^{2} \varphi}{\partial x^{2}}=0
$$

while the regeneration of action potentials at the nodes of Ranvier is described by the following ordinary differential equation:

$$
b \frac{\partial \varphi}{\partial t}+f(\varphi)=0
$$

By using the last equation and Figure 20, as well as the same line of reasoning as in the derivation of formulas (3.28) and (3.29), we find the follow expressions for $f(\varphi)$ :

$$
\begin{gathered}
f(\varphi)=f_{N a^{+}}(\varphi)+f_{K^{+}}(\varphi), \\
f_{N a^{+}}(\varphi)=-b \frac{\varphi_{\max }-\varphi_{\text {rest }}}{t_{2}-t_{1}} u\left(\varphi-\varphi_{\text {rest }}\right) \hat{\gamma}_{N a^{+}}(\varphi), \\
f_{K^{+}}(\varphi)=b \frac{\varphi_{\max }-\varphi_{\text {rest }}}{t_{3}-t_{2}} \hat{\gamma}_{K^{+}}(\varphi),
\end{gathered}
$$

where $\hat{\gamma}_{N a^{+}}(\varphi)$ and $\hat{\gamma}_{K^{+}}(\varphi)$ are the rectangular hysteresis loop shown in Figures 17 and 16, respectively. It is clear that $f_{\mathrm{Na}^{+}}(\varphi)$ is the component of $f(\varphi)$ representing the sodium channel currents which are responsible for a steep upstroke in the action potential, while $f_{K^{+}}(\varphi)$ represents the potassium channel currents responsible for the reversal branch of the action potential. It must be remarked that the nature of potassium channels at 
the node of Ranvier is still a matter of discussion. There exists the point of view that these are special calciumactivated potassium channels. Another point of view is that the action potential repolarization is achieved due to strong leakage channels, and this explains the absence of any hyperpolarization in action potentials at nodes of Ranvier.

Between the nodes of Ranvier, the dynamics of electric potential is described by equation (3.30). This is a linear diffusion equation. It is known that for such an equation, the speed of propagation of zero front is infinite. In this sense, it does not describe any wave propagation effects. However, it does describe fast-in-time spatial variation of the potential $\varphi$ due to diffusion. It is clear from equation (3.30) that the smaller the coefficient $b$, the faster the process of diffusion. The smallness of $\mathrm{b}$ is achieved by decreasing the per unit surface capacitance $C$ (see formula (3.11)) through the myelination of axon. This leads to the following picture of saltatory signal conduction in myelinated axons. Namely, the potential perturbations caused by the action potential at a node of Ranvier is transmitted through a fast diffusion process to another node of Ranvier where this perturbation leads to regeneration of the action potential and its further transmission to yet another node of Ranvier. In this way, the transmission of the action potential occurs through multiple repetitions of the described process of "hopping" of the action potential from node to node.

In our previous discussion, we used triangular shape approximations of action potentials, which appreciably simplified the solution of the inverse problem of determination of $f(\varphi)$. This solution clearly reveals the hysteretic nature of macroscopic sodium and potassium currents, which are expressed in terms of the rectangular loops shown in Figures 16, 17 and 19. Similar calculations can be performed for more realistic shapes of action potentials and they will lead to hysteresis loops with gradual (instead of vertical) transitions between two states. The possibility of such calculation is especially evident from equation (3.31) for generation of the action potential at the nodes of Ranvier. Similar calculations can also be carried out by using equation (3.19), however, they are more involved.

\section{Conclusion}

Since ion channel proteins have many conformational (metastable) states they exhibit hysteresis. In this context, it is shown that rectangular hysteresis loops play an important role in understanding and modeling the electrical behavior of ion channels. It is asserted that metastable states of the ion channels are responsible for the non-Markovian stochastic nature of binary ion channel recordings. Accordingly, it is proposed that the stochastic single channel recordings can be modeled as the random outputs of rectangular hysteresis loops driven by stochastic processes. Based on this approach, it is shown that the problem can be mathematically treated as an exit problem for stochastic processes or by using the theory of stochastic processes on graphs. Some of the key mathematical formulations of stochastic processes on graphs applicable to the modeling of ion channels are presented. Finally, it is shown in the paper that the collective action of sodium and potassium channels responsible for the generation and propagation of action potentials exhibit hysteresis. This is accomplished by using the inverse problem approach to the nonlinear Hodgkin-Huxley diffusion equation. In this way, it is shown that hysteretic collective ion channel properties can be characterized in terms of observed action potentials. Analysis is presented for the cases of non-myelinated and myelinated axons, with Ranvier nodes.

\section{Appendix A. BAsic NeUroscience BACKGRound}

Neurons, and cells in general, are defined by their membranes which separate their interior from the exterior environment. Membranes are formed by phospholipid molecules. These molecules have two-long hydrophobic ("water-repelling") fatty acid tails and hydrophilic ("water-attracting") phosphorous containing heads. In water, these molecules align themselves in double layers in such a way that hydrophobic fatty tails are inside these layers (i.e. away from water), while hydrophilic heads point outwards into the water on both exterior and interior sides of neuron boundaries formed by these double layers. These lipid bilayers are of 3 to $5 \mathrm{~nm}$ in thickness, and they represent insulating barriers separating conducting water solutions on interior and exterior sides of neurons. 


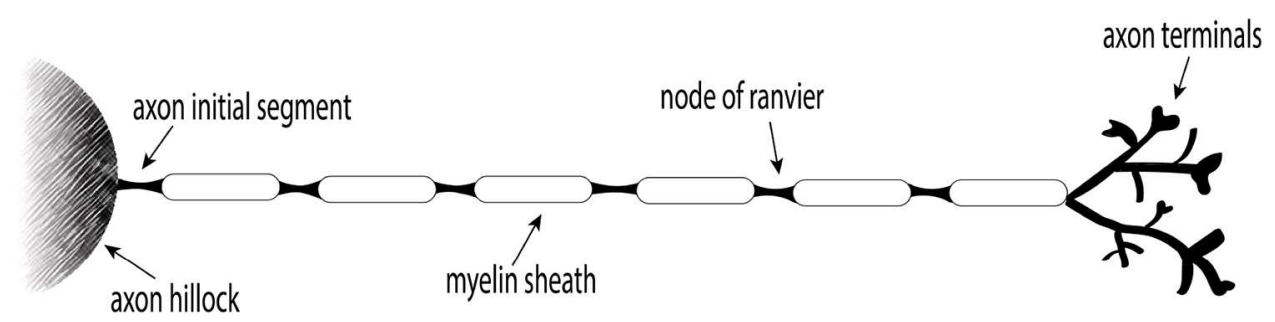

Figure A.1. Schematic of an axon.

Neurons reside in external conducting ionic solutions. The concentrations of the various ions (such as, sodium, potassium, calcium, etc.) are such that there is a concentration gradient across the neuron membrane. The ion gradients are formed as a result of the action of special protein molecules embedded in the membrane of the cells (ion pump). Ion pumps move ions against their concentration gradients. This means that pumps execute the active ion transport towards locations of higher concentration. This active transport requires some energy which is usually supplied by special ATP (Adenosine Triphosphate) molecules. As a result of the resulting concentration gradient across the cell membrane, a potential difference is established where the potential inside the cell is more negative with respect to the extracellular space, typically about $-70 \mathrm{mV}$.

This potential difference is referred to as the "rest potential". Neurons transmit information by generating and transmitting action potentials which are typically a sequence of pulses about $100 \mathrm{mV}$ high and are formed by the local injection and extraction of ions across the neuronal membrane. This action is generally achieved by specific protein molecules which form ion-channels embedded within the cellular membrane. These channels are temporarily opened and closed to allow the flow of ions from areas of high concentration to low concentration. For instance, sodium ion channels allow the inward flow of ions, whereas the potassium channels allow the outward flow of ions. This inward and outward flow results in local potential spikes that propagate along the axon of neurons and the information is transmitted to other connected neurons by synapses. The ion channels play the central role in this electrical signaling in neurons, and they are discussed in some detail in Section 2.

In neurons, the three main structures can be identified as soma (cell body), axon and dendrites. Anatomically, an axon is a tubular type protrusion emanating at the axon hillock site of soma and extending away for long distances. A schematic of axon is presented in Figure A.1. Long axons of the vertebrate neurons system are usually covered with myelin. This is a tight many layer wrapping of insulating glial cell membrane which is usually called myelin sheath. This insulation results in faster signal speed as well as the reduction of signal decay. About every millimeter, the myelin sheath is periodically interrupted by exposing naked excitable axon membrane to the extracellular fluids. These unmyelinated spots of axons are called nodes of Ranvier, and they are about $1 \mu \mathrm{m}$ long. Myelin insulation prevents ions from entering or leaving the axon along myelinated segments. This implies that the ion-transport through axon membrane is strongly localized at the nodes of Ranvier. The realization of this localization of ion transport is achieved due to high concentration of ion channels in axon membrane at nodes of Ranvier.

Another location with high concentration of ion-channels is the axon initial segment. It is relatively thick, unmyelinated part of the axon that emanates directly from the cell body. It is about $25 \mu \mathrm{m}$ in length, and at this site the initiation of action potential occurs. This action potential moves very fast (leaps) through myelinated segments of axon being sequentially regenerated and properly enhanced at the nodes of Ranvier. This "hopping" of action potential from one node of Ranvier to another is known as "saltatory" conduction. At the end, an axon is divided into many unmyelinated branches, which serve as axon terminals. These terminals form synaptic connections with dendrites of other neurons. It is worthwhile to mention that axons of neuron cells in invertebrates are unmyelinated. This results in the different physical mechanism of action potential propagation. This mechanism is based on nonlinear diffusion which is supported by relatively uniform (i.e. homogeneous) distribution of ion-channels along axon membranes. This nonlinear diffusion mechanism has been extensively (experimentally and theoretically) studied in the case of squid giant axons. This study was 
initiated by Alan Hodgkin and Andrew Huxley in Cambridge, England in the middle of the last century. This resulted in the creation of Hodgkin-Huxley model of action potential generation and propagation. This model is widely regarded as one of the greatest achievements in the neural science and has been very influential in the subsequent research in this field.

\section{Appendix B. Noise in Hysteretic Systems AND STOCHAstiC PROCESSES ON GRAPHS}

Here we discuss the mathematical framework of hysteresis driven by a continuous time and continuous sample diffusion process. The mathematical treatment of this problem is reduced to the so-called "exit problem" for diffusion processes [18]. Although the concept of random switching as an exit problem is quite transparent from the physical point of view, its mathematical implementation is somewhat complicated. It turns out that the mathematical treatment can be simplified by using an entirely different approach based on theory of stochastic processes on graphs. This theory has recently been developed and applied to the study of random perturbations of Hamiltonian dynamical systems [19]-[21]. The main purpose of this Appendix is to demonstrate that the mathematical machinery of this theory is naturally suitable for the analysis of random output processes of hysteretic systems. The discussion presented below closely follows the paper [21].

This discussion is based on the following simple fact. The output of the rectangular hysteresis loop $i_{t}=\hat{\gamma}_{\alpha \beta} x_{t}$ is a random binary process. This process is not Markovian. However, the two-component process $\vec{y}_{t}=\left(\begin{array}{l}i_{t} \\ x_{t}\end{array}\right)$ is Markovian. This is because the rectangular loop operators describe hysteresis with local memory. This means that joint specifications of current values of input and output uniquely define the states of this hysteresis. The two-component process $\vec{y}_{t}$ is defined on the four-edge graph shown in Figure B.1, which corresponds to rectangular loop $\hat{\gamma}_{\alpha \beta}$ with output values of \pm 1 . The binary process $i_{t}$ presumes constant values on each edge $I_{k},(k=1,2,3,4)$ of this graph.

In this Appendix, we consider symmetric rectangular loops with output values \pm 1 , because this simplifies the mathematical treatment of the problem. However, the presented analysis can be used for nonsymmetric rectangular loops shown in Figure 14. This is because the latter rectangular loop can be obtained from the symmetric loop by shifting its output by one and subsequently scaling it by one half.

The two component Markovian process $\vec{y}_{t}$ can be characterized by the transition probability density:

$$
\rho\left(\vec{y}, t \mid \vec{y}_{0}, t_{0}\right)
$$

defined on all edges of the above graph. This justifies the following equations:

$$
\left.\rho\left(\vec{y}, t \mid \vec{y}_{0}, t_{0}\right)\right|_{\vec{y} \in I_{k}}=\rho^{(k)}\left(x, t \mid \vec{y}_{0}, t_{0}\right)
$$

It is apparent that

$$
\begin{aligned}
& \rho^{(1)}=\rho, \text { for } x \leq \beta, \\
& \rho^{(4)}=\rho, \text { for } x \geq \alpha,
\end{aligned}
$$

and

$$
\rho^{(2)}+\rho^{(3)}=\rho, \text { for } \beta \leq x \leq \alpha,
$$




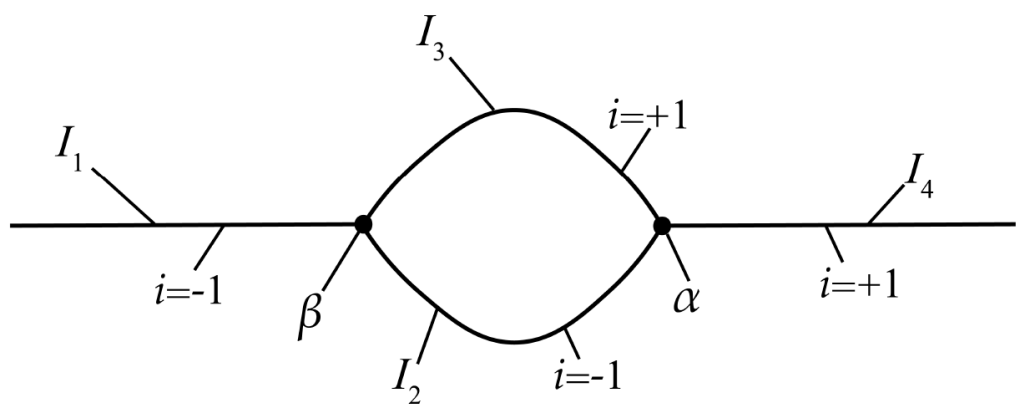

Figure B.1. Triangular shaped action potential.

where $\rho$ is the transition probability density of the process $\vec{y}_{t}$, which is assumed to be known. It is also clear that on each edge of the graph the following forward Kolmogorov equation is valid

$$
\frac{\partial \rho^{(k)}}{\partial t}=\frac{1}{2} \frac{\partial^{2}}{\partial x^{2}}\left[\sigma^{2}(x) \rho^{(k)}\right]-\frac{\partial}{\partial x}\left[b(x) \rho^{(k)}\right] .
$$

Furthermore, the Markovian nature of the process $\vec{y}_{t}$ on the entire graph implies certain boundary conditions for $\rho^{(k)}$ at graph vertices. It can be shown that these vertex boundary conditions impose continuity of transition probability density when the transition through a vertex form one graph edge to another occurs without rectangular loop switching, and zero boundary condition is imposed on the third graph edge connected to this vertex. Namely, for edge $I_{3}$ we have:

$$
\left.\rho^{(3)}\right|_{x=\beta}=0,
$$

while

$$
\left.\rho^{(3)}\right|_{x=\alpha}=\left.\rho\right|_{x=\alpha} .
$$

Similarly, for edge $I_{2}$ we find:

$$
\left.\rho^{(2)}\right|_{x=\beta}=\left.\rho\right|_{x=\beta},
$$

while

$$
\left.\rho^{(2)}\right|_{x=\alpha}=0
$$

In addition, the so-called probability current

$$
J_{k}(x)=-\frac{\sigma^{2}(x)}{2} \frac{\partial \rho^{(k)}}{\partial x}+b(x) \rho^{(k)}(x)
$$

must be conserved at each vertex. According to formulas (B.7)-(B.11), this implies the following boundary conditions:

$$
\begin{aligned}
& \left.\frac{\partial \rho}{\partial x}\right|_{x=\beta}=\left.\frac{\partial \rho^{(2)}}{\partial x}\right|_{x=\beta}+\left.\frac{\partial \rho^{(3)}}{\partial x}\right|_{x=\beta}, \\
& \left.\frac{\partial \rho}{\partial x}\right|_{x=\alpha}=\left.\frac{\partial \rho^{(2)}}{\partial x}\right|_{x=\alpha}+\left.\frac{\partial \rho^{(3)}}{\partial x}\right|_{x=\alpha},
\end{aligned}
$$


Furthermore, the following initial condition is valid

$$
\rho\left(x, 0 \mid x^{\prime}, 0\right)=\delta_{k k^{\prime}} \delta\left(x-x^{\prime}\right) .
$$

Equations (B.6) along with boundary conditions (B.7)-(B.10) and (B.12)-(B.13) as well as initial condition (B.14) completely define the dynamics of the transitional probability density for the two component Markovian process $\vec{y}_{t}$ on the graph shown in Figure B.1. The solutions to the above initial-boundary value problem can be found in terms of parabolic cylinder functions and their Laplace transforms in the case when $x_{t}$ is the OrnsteinUhlenbeck. It turns out that simple analytical results can be obtained for stationary densities $\rho_{\mathrm{st}}^{(3)}$ and $\rho_{\mathrm{st}}^{(2)}$. In this case we have to deal with the following boundary value problem for ordinary differential equations: find the solution of equation

$$
\frac{1}{2} \frac{\mathrm{d}^{2}}{\mathrm{~d} x^{2}}\left[\sigma^{2}(x) \rho_{\mathrm{st}}^{(3)}(x)\right]-\frac{\mathrm{d}}{\mathrm{d} x}\left[b(x) \rho_{\mathrm{st}}^{(3)}(x)\right]=0
$$

subject to the boundary conditions

$$
\rho_{\mathrm{st}}^{(3)}(\beta)=0,
$$

while

$$
\rho_{\mathrm{st}}^{(3)}(\alpha)=\rho_{\mathrm{st}}(\alpha)
$$

The above boundary conditions follow from formulas (B.7) and (B.8).

Having solved the boundary value problem (B.15)-(B.17) for $\rho_{\mathrm{st}}^{(3)}, \rho_{\mathrm{st}}^{(2)}$ can be found from formula (B.5) as

$$
\rho_{\mathrm{st}}^{(2)}(x)=\rho_{\mathrm{st}}(x)-\rho_{\mathrm{st}}^{(3)}(x) .
$$

The last equation implies that the boundary conditions (B.12) and (B.13) will be satisfied. The analytical solution to the boundary value problem (B.15)-(B.17) can be found for any stationary diffusion process $x_{t}$. However, below we present this solution only for the important case of the Ornstien-Uhlenbeck process. By integrating equation (B.15) for this process, we find:

$$
\frac{\sigma^{2}}{2} \frac{\mathrm{d} \rho_{\mathrm{st}}^{(3)}}{\mathrm{d} x}+k x \rho_{\mathrm{st}}^{(3)}(x)=C=\text { const. }
$$

We look for the solution of equation (B.19) in the form

$$
\rho_{\mathrm{st}}^{(3)}(x)=\rho_{\mathrm{st}}(x) \varphi(x)
$$

where $\rho_{\mathrm{st}}(x)$ is the Gaussian stationary distribution of the Ornstein-Uhlenbeck process, while $\varphi(x)$ must be determined. By substituting formula (B.20) into equation (B.19), we find:

$$
\frac{\sigma^{2}}{2} \rho_{\mathrm{st}}(x) \frac{\mathrm{d} \varphi(x)}{\mathrm{d} x}+\varphi(x)\left[\frac{\sigma^{2}}{2} \frac{\mathrm{d} \rho_{\mathrm{st}}(x)}{\mathrm{d} x}+k x \rho_{\mathrm{st}}(x)\right]=C .
$$


For the stationary Ornstein-Uhlenbeck process, the probability current (B.11) is equal to zero, which means that

$$
\frac{\sigma^{2}}{2} \frac{\mathrm{d} \rho_{\mathrm{st}}(x)}{\mathrm{d} x}+k x \rho_{\mathrm{st}}(x)=0 .
$$

From the last two equations, we find

$$
\frac{\sigma^{2}}{2} \rho_{\mathrm{st}}(x) \frac{\mathrm{d} \varphi(x)}{\mathrm{d} x}=C
$$

By integrating equation (B.23) and taking into account boundary condition (B.16) and formula (B.20), we obtain:

$$
\varphi(x)=\frac{2 C}{\sigma^{2}} \int_{\beta}^{x} \frac{\mathrm{d} y}{\rho_{\mathrm{st}}(y)} .
$$

From boundary condition (B.17) and formula (B.20), it follows that

$$
\varphi(\alpha)=1
$$

From equations (B.24) and (B.25), we find:

$$
1=\frac{2 C}{\sigma^{2}} \int_{\beta}^{\alpha} \frac{\mathrm{d} y}{\rho_{\mathrm{st}}(y)}
$$

and

$$
\frac{2 C}{\sigma^{2}}=\frac{1}{\int_{\beta}^{\alpha} \frac{\mathrm{d} y}{\rho_{\mathrm{st}}(y)}} .
$$

Now, by recalling formula (B.24), we derive

$$
\varphi(x)=\frac{\int_{\beta}^{x} \frac{\mathrm{d} y}{\rho_{\mathrm{st}}(y)}}{\int_{\beta}^{\alpha} \frac{\mathrm{d} y}{\rho_{\mathrm{st}}(y)}},
$$

which according to equation (B.20) leads to

$$
\rho_{\mathrm{st}}^{(3)}(x)=\rho_{\mathrm{st}}(x) \frac{\int_{\beta}^{x} \frac{\mathrm{d} y}{\rho_{\mathrm{st}}(y)}}{\int_{\beta}^{\alpha} \frac{\mathrm{d} y}{\rho_{\mathrm{st}}(y)}},
$$

where the analytical expression for $\rho_{\mathrm{st}}(x)$ is given by formula (B.26). 
By using the last formula, the stationary expected value of the random binary process $i_{t}=\hat{\gamma}_{\alpha \beta} x_{t}$ and its variance $\sigma_{i_{t}}^{2}$ can be computed. Indeed, it is clear that

$$
\overline{i_{t}}=E^{\mathrm{st}}\left\{\hat{\gamma}_{\alpha \beta} x_{t}\right\}=P_{\mathrm{st}}\left\{i_{t}=1\right\}-P_{\mathrm{st}}\left\{i_{t}=-1\right\} .
$$

Since

$$
P_{\text {st }}\left\{i_{t}=1\right\}+P_{\text {st }}\left\{i_{t}=-1\right\}=1 \text {, }
$$

we find

$$
\overline{i_{t}}=2 P_{\text {st }}\left\{i_{t}=1\right\}-1 .
$$

On the other hand,

$$
P_{\mathrm{st}}\left\{i_{t}=1\right\}=\int_{\beta}^{\alpha} \rho_{\mathrm{st}}^{(3)}(x) \mathrm{d} x+\int_{\alpha}^{\infty} \rho_{\mathrm{st}}(x) \mathrm{d} x .
$$

Consequently,

$$
\overline{i_{t}}=2\left[\int_{\beta}^{\alpha} \rho_{\mathrm{st}}^{(3)}(x) \mathrm{d} x+\int_{\alpha}^{\infty} \rho_{\mathrm{st}}(x) \mathrm{d} x\right]-1 .
$$

As soon as $\overline{i_{t}}$ is computed by using formulas (B.29) and (B.34), variance $\sigma_{i_{t}}^{2}$ can be calculated as well. Indeed, by using the well-known relation

$$
\sigma_{i_{t}}^{2}=\bar{i}_{t}^{2}-\left(\overline{i_{t}}\right)^{2}
$$

and the fact that

$$
i_{t}^{2}=1
$$

we derive

$$
\sigma_{i_{t}}^{2}=1-\left(\overline{i_{t}}\right)^{2}
$$

The last formula along with equation (B.34) can be used for computations of variance $\sigma_{i_{t}}^{2}$.

\section{REFERENCES}

[1] W.A. Catterall, Structure and function of voltage-gated sodium channels at atomic resolution. Exp. Physiol. 99 (2014) 1-26.

[2] V.A. Chinarov, Y.B. Gaididei, V.N. Kharkyanen and S.P. Sitko, Ion pores in biological membranes as self-organized bistable systems. Phys. Rev. A 46 (1992) 5232-5241.

[3] P.S. Churchland and T.J. Sejnowski, The Computational Brain. A Bradford Book. The MIT Press (1992).

[4] F. Crick, Memory and molecular turnover. Nature 312 (1984) 101.

[5] P. Dayan and L.F. Abbott, Theoretical Neuroscience. The MIT Press (2001).

[6] H. Flyvbjerg, E. Gudowska-Nowak, P. Chrsitophersen and P. Bennekou, Modeling hysteresis observed in the human erythrocyte voltage-dependent cation channel. Acta Phys. Pol. Ser. B 43 (2012) 2117-2140.

[7] M.I. Freidlin, Markov Processes and Differential Equations: Asymptotic Problems. Birkhäuser-Berlin, Berlin (1996).

[8] M.E. Freidlin and A.D. Wentzell, Diffusion Processes on Graphs and the Averaging Principle. Ann. Probab. 21 (1993) $2215-2245$. 
[9] M.I. Freidlin, I.D. Mayergoyz and R. Pfeiffer, Noise in hysteretic systems and stochastic processes on graphs. Phys. Rev. E 62 (2000) 1850-1855.

[10] A. Fulinski, Z. Grzywna, I. Mellor, Z. Siwy and P.N.R. Usherwood, Non-Markovian character of ionic current fluctuations in membrane channels. Phys. Rev. E 58 (1998) 919-924.

[11] C.R. Gallistel and A.P. King, Memory and the Computational Brain. Wiley-Blackwell (2009).

[12] B. Hille, Ion Channels of Excitable Membranes. Third Edition, Sinauer Associates, Inc. (2001).

[13] J.J.B. Jack, D. Noble and R.W. Tsien, Electric Current Flow in Excitable Cells. Oxford University Press (1983).

[14] C. Koch, Biophysics of Computation. Oxford University Press (1999).

[15] C.E. Korman and I.D. Mayergoyz, Switching as an Exit Problem. IEEE Trans. Magn. 31 (1995) 3545-3547.

[16] R. Männikkö, S. Pandey, H.P. Larsson and F. Elinder, Hysteresis in the voltage dependence of HCN channels: conversion between two modes affects pacemaker properties. J. General Physiol. 125 (2005) 305-326.

[17] I.D. Mayergoyz, W.W. Destler and F.P. Emad, Application of intense relativistic electron beams to the switching of high currents in high power electrical networks. J. Appl. Phys. 53 (1982) 7189-7194.

[18] R. Meagher, Memory and molecular turnover,' 30 years after inception. Epigen. Chrom. 7 (2014) 37.

[19] V. Nache, T. Eick, E. Schulz, R. Schmauder and K. Benndorf, Hysteresis of ligand binding in CNGA2 ion channels. Nat. Commun. 4 (2013) 1-9.

[20] E. Neher and B. Sakmann, Single-channel currents recorded from membrane of denervated frog muscle fibres. Nature 260 (1976) 799-802.

[21] M.A. Pustovoit, A.M. Berezhkovskii and S.M. Bezrukov, Analytical theory of hysteresis in ion channels: two-state model. J. Chem. Phys. 125 (2006) 194907.

[22] C. Tilegenova, D.M. Cortes and L.G. Cuello, Hysteresis of KcsA potassium channel's activation-deactivation gating is caused by structural changes at the channel's selectivity filter. PNAS 114 (2017) 3234-3239.

[23] J. Timmer and S. Klein, Testing the Markov condition in ion channel recordings. Phys. Rev. E 55 (1997) $3306-3311$.

[24] H.C. Tuckwell, Vols. 1 and 2 of Introduction to Theoretical Neurobiology. Cambridge University Press (1988).

[25] C.A. Villalba-Galea, Hysteresis in voltage-gated channels. CHANNELS 11 (2017) 140-155. 